\title{
MRSA und Datenschutz - eine Paranoia schärft ihr Profil!
}

\author{
Heinz-Michael Just
}

Die Männer und Frauen im Einwegoverall, Atemschutz und Kopfbedeckung, die ins Stationszimmer treten, bringen nicht etwa einen Patienten mit Schweinegrippe oder SARS, nein, sie holen einen alten Menschen, der in sein Heim zurückverlegt werden soll. Was er hat? Nichts Besonderes; seine chronische Erkrankung wurde behandelt und sein Ulcus versorgt. Aber kurz vor der Verlegung wurde noch ein Abstrich in die Bakteriologie geschickt und zurück kam der Befund: MRSA-Besiedlung.

Bräuchte der Patient keine Transportbetreuung, würde er mit dem Taxi fahren, mit der U-Bahn, mit dem Bus und kein Mensch würde ihm seine Staphylokokken ansehen, denen es gelungen ist, sich trotz Anwesenheit von Oxacillin weiter zu vermehren. Aber so schauen alle fasziniert und ängstlich, wer hier denn so gefährlich ist. Und das Heim wurde bereits darüber informiert, dass der Ankömmling MRSA-positiv ist - so, wie es im Ratgeber „Infektionskrankheiten - Merkblätter für Ärzte“ des Robert-Koch-Instituts (RKI) steht: „Dringend erforderlich ist hierbei die vertrauensvolle Zusammenarbeit zwischen Heimleitung, betreuenden Ärzten und verlegender Einrichtung. Dies gilt besonders für die gegenseitige Vorabinformation über den Besiedlungsstatus von zu verlegenden MRSA-positiven Bewohnern/Patienten“. Auch die Deutsche Gesellschaft für Krankenhaushygiene (DGKH) empfiehlt: „Die Zieleinrichtung und der Krankentransportdienst sind über die MRSA-Besiedlung/-infektion bei dem Patienten rechtzeitig vorab zu informieren ... “ Und in den offiziellen Verlautbarungen eines Bundeslandes steht bei „Maßnahmen bei MRSA für Rettungs- und Krankentransportdienste“ unter „Sicherung des Informationsflusses“ zu lesen: „Die Einsatzkräfte müssen über MRSA und über die spezifische Sachlage beim zu transportierenden Patienten informiert sein“ und weiter: „Vor dem Transport eines MRSA-positiven Patienten ist der betreffende Rettungs- und Transportdienst von der anfordernden Stelle über den bestehenden Sachverhalt zu informieren.“

Wirklich?
Wir haben ein Datenschutzgesetz und unterliegen der ärztlichen Schweigepflicht ( $\$ 203$ StGB: I. Wer unbefugt ein fremdes Geheimnis ... offenbart, das ihm als Arzt, ... oder Angehörigen eines anderen Heilberufs ... anvertraut worden oder sonst bekannt geworden ist, wird mit Freiheitsstrafe bis zu einem Jahr oder Geldstrafe bestraft ...). Beides sind hoch angesiedelte Rechtsgüter, die nur bei besonderer Gefahrenlage übertreten werden dürfen. Ist eine solche bereits bei einem Bakterienstamm gegeben, der gegen eine von mehreren Antibiotikaklassen resistent geworden ist? Und reicht dafür sogar bereits eine Besiedlung? Ich meine nein, denn ein MRSA ist primär nicht virulenter oder pathogener als ein MSSA - wir hatten über lange Zeit lediglich nur ein Antibiotikum als Therapieempfehlung und das galt als nur bakteriostatisch wirksam, toxisch und war zudem teuer. Von daher waren die Beunruhigung und die daraus resultierenden Empfehlungen zur Vermeidung einer Weiterverbreitung in Krankenhäusern zu jener Zeit verständlich, nicht jedoch die sie damals schon begleitende Hysterie außerhalb!

Auf welcher Rechtsgrundlage basiert also die Empfehlung des RKI, die Schweigepflicht in solchen Fällen zu brechen? Die Heimleitung ist kein Arzt - und schon gar nicht der des Patienten! Auf welcher Rechtsgrundlage beruht die Empfehlung des zitierten Bundeslandes? Hat der Patient den Stationsarzt vorher von der Schweigepflicht entbunden - was bei einer Information außerhalb des Arztbriefs, also insbesondere gegenüber „dem Krankentransport“, „einer Institution“, der „Heimleitung“, der Pflegekraft im Heim, die Voraussetzung wäre? Wohl kaum. In diesem Zusammenhang sei auch auf eine Erinnerung im Ärzteblatt vom 4. 2. 2005 verwiesen, wo es heißt: „Cave (Häufiger Irrtum): Schweigepflicht gilt auch gegenüber den ärztlichen Kollegen, die nicht in die Behandlung bzw. das Arzt-Patienten-Verhältnis einbezogen sind. "Also nur der behandelnde Arzt und nicht irgendein, wenn auch „zuständiger“ Heimarzt darf informiert werden. Ein Aspekt, der im Rahmen der momentanen MRSA-Netzwerk-Euphorie in Deutschland im Auge behalten werden sollte! 
Auf den Internetseiten der AWMF (Arbeitsgemeinschaft der wissenschaftlichen Medizinischen Forschungsgesellschaften) steht nunmehr gar unter „Maßnahmen beim Auftreten multiresistenter Erreger (MRE)“: „Es ist sicher zu stellen, dass vorbehandelnde und nachbehandelnde Personen/Institutionen informiert werden. Diese sind bei Nachweis von MRE sofort zu informieren, damit entsprechende Maßnahmen auch dort unverzüglich durchgeführt werden können (...).“Also künftig Missachtung der ärztlichen Schweigepflicht auch bei anderen Bakteriennachweisen, die als „multiresistent“ eingestuft werden - ohne dass überhaupt klar ist, wie eine „Multiresistenz" definiert ist [1]? Ein Wildwuchs an Rechtsübertretungen durch behandelnde (Krankenhaus-)Ärzte auf der Basis fachlicher bzw. sogar öffentlich-rechtlicher jedoch inkorrekter Empfehlungen?

Es scheint dringend an der Zeit, die zunehmende Resistenzentwicklung von Bakterien als das zu empfinden, was sie ist: eine von uns geschaffene Bedrohung. Es ist richtig darauf hinzuweisen alles zu tun, einer solchen Gefährdung zu begegnen. Dies kann aber nicht primär Isolierung aller „positiven“ Personen heißen oder gar allgemeine Offenlegung einer Besiedlung, was letztlich in der öffentlichen Wahrnehmung zunehmend einer Stigmatisierung gleichkommt, sondern muss verantwortungsbewusstes Handeln aller Beteiligten unter Beachtung aller zu schützenden Rechtsgüter zur Folge haben, wozu auch eine geregelte Informationsweitergabe gehört. Der Fokus hat sich deshalb in 1. Linie auf den Kampf gegen den Missbrauch von Antibiotika in allen Bereichen zu richten, um weitere Resistenzentstehungen zu vermeiden, und nicht darauf, wie dann mit diesen Resistenzen bzw. mit den Betroffenen umzugehen ist.

MRSA-Netzwerke zu gründen ist in Deutschland derzeit in Mode aber nur dann sinnvoll, wenn in der Sache Fortschritte erzielt werden - und zwar evidenzbasiert! Bayern hat eine Landesarbeitsgemeinschaft Multiresistente Erreger (LARE; [2]) gegründet mit Arbeitsgruppen zu im Alltag drängenden Fragen, darunter auch einer AG „Informationsweitergabe“. Dort arbeitet man an einer Lösung, bei Verlegungen so zu informieren, dass die ärztliche Schweigepflicht gewahrt bleibt und trotzdem deutlich wird, dass gegebenenfalls zusätzliche Vorkehrungen zu treffen sind und die aufnehmende Einrichtung dafür sorgen muss, die z.B. nach der KRINKO-Empfehlung für die Heime [3] angeratene Risikoabschätzung unverzüglich vornehmen zu können. Wichtig ist in diesem Zusammenhang, über entsprechende Institutionen (z. B. Landesärztekammern, Kassenärztliche Vereinigungen, Gesundheitsämter) niedergelassene Kolleginnen und Kollegen, aber auch andere involvierte Berufsgruppen im Umgang mit antibiotikaresistenten Bakterien zu schulen, denn hier sind noch erhebliche Defizite offenkundig. Eine lohnende Aufgabe auch für die Netzwerke!

Die Notwendigkeit hierfür sei an einem Beispiel aus Süddeutschland belegt: Bei einer chronisch erkrankten Schülerin wird eine MRSA-Besiedlung offenkundig. Daraufhin wird von der Schule ernsthaft überlegt, ob nicht eine Unterrichtung des Kindes zuhause angezeigt sei. Pausenkontakte mit andern Kindern werden infrage gestellt, die Teilnahme an einem Landschulaufenthalt nur unter der Bedingung ermöglicht, dass ein Elternteil mitfährt und täglich das Bett frisch bezieht und die Wäsche in einem Plastiksack sammelt... Dem zuständigen Gesundheitsamt ist es nach eigenen Angaben in monatelangem „Dialog“ mit der Schule (noch) nicht gelungen, einen rationalen Umgang mit dieser Schülerin zu erreichen. Paranoia wie sie im Lehrbuch stehen könnte!

Deshalb wird in einem der nächsten Hefte eine erfahrene Justiziarin einer großen Klinik dieses Thema ausführlich behandeln.

\section{Literatur}

1 Multidrug-resistant (MDR), extensively drug-resistant (XDR) and pandrug-resistant (PDR) bacteria in healthcare settings. Expert proposal for a standardized international terminology. ECDC Entwurf 2010 (www.escmid.org)

2 Im Internet: http://www.Igl.bayern.de/

3 Bundesgesundheitsbl - Gesundheitsforsch Gesundheitsschutz 2005; 48: 1061 - 1080 